\title{
Perirhinal cortex is necessary for acquiring, but not for retrieving object-place paired association
}

\author{
Yong Sang Jo ${ }^{1}$ and Inah Lee ${ }^{2,3}$ \\ ${ }^{1}$ Department of Psychology, University of Washington, Seattle, Washington 98195, USA; ${ }^{2}$ Department of Brain and Cognitive \\ Sciences, Seoul National University, Gwanak-ro, Gwanak-gu, Seoul 151-742, Korea
}

\begin{abstract}
Remembering events frequently involves associating objects and their associated locations in space, and it has been implicated that the areas associated with the hippocampus are important in this function. The current study examined the role of the perirhinal cortex in retrieving familiar object-place paired associates, as well as in acquiring novel ones. Rats were required to visit one of two locations of a radial-arm maze and choose one of the objects (from a pair of different toy objects) exclusively associated with a given arm. Excitotoxic lesions of the perirhinal cortex initially impaired the normal retrieval of object-place paired-associative memories that had been learned presurgically, but the animals relearned gradually to the level of controls. In contrast, when required to associate a novel pair of objects with the same locations of the maze, the same lesioned rats were severely impaired with minimal learning, if any, taking place throughout an extensive testing period. However, the lesioned rats were normal in discriminating two different objects presented in a fixed arm in the maze. The results suggest that the perirhinal cortex is indispensable to forming discrete representations for object-place paired associates. Its role, however, may be compensated for by other structures when familiar object-place paired associative memories need to be retrieved.
\end{abstract}

Remembering an event in space often requires associating objects and their locations. Associating object and place information into a unitary event representation is believed to be a foundation of episodic memory (Cahusac et al. 1989; Gaffan 1994; Davachi 2006). It has been suggested that the hippocampus and its associated regions in the medial temporal lobe (MTL) are essential in this cognitive process, and amnesic patients with damage in the MTL structures exhibit severe deficits in associating object and place information (Smith and Milner 1981; Vargha-Khadem et al. 1997; Stepankova et al. 2004). Animal models produced by localized lesions in the hippocampus and other MTL structures also support the idea by showing that the lesioned animals are impaired in associating objects and places (Parkinson et al. 1988; Gaffan and Parker 1996; Sziklas et al. 1998; Bussey et al. 2001; Gilbert and Kesner 2003, 2004; Malkova and Mishkin 2003; Lee et al. 2005; Bachevalier and Nemanic 2008; Kesner et al. 2008; Lee and Solivan 2008). Although the theoretical importance of the MTL structures in object-place association has been well acknowledged, specific contributions of the MTL structures in object-place associative memory are poorly understood. The current study examined the role of the perirhinal cortex, one of the extra hippocampal regions in the MTL, using a behavioral paradigm previously shown to be dependent on the intact hippocampus (Lee and Solivan 2008).

The literature suggests that the role of the hippocampus in the object-place paired-associate task is to put together object and place information into a unified and distinct event representation. It has been suggested that spatial information and nonspatial information (such as object information) may be streamed into the hippocampus in a relatively segregated fashion, the former information mostly fed through the medial entorhinal cortex to the hippocampus via the postrhinal cortex and the latter being fed through the lateral entorhinal cortex via the perirhinal cortex (Mishkin et al. 1997; Suzuki et al. 1997; Burwell 2000; Fyhn et al. 2004; Witter and Amaral 2004; Hafting et al. 2005; Hargreaves

\footnotetext{
${ }^{3}$ Corresponding author.
}

E-mail inahlee@snu.ac.kr; fax 82-2-8719129.

Article is online at http://www.learnmem.org/cgi/doi/10.1101//m.1620410. et al. 2005; Furtak et al. 2007; Kerr et al. 2007). In our previous study (Lee and Solivan 2008) in which rats were required to discriminate rewarding versus nonrewarding pairs of similar object-place paired associates, the hippocampal lesioned rats demonstrated severe and irrecoverable deficits. The results from the study not only corroborate the long-held view that the hippocampus associates object and place information, but also demonstrate that the hippocampus is critical for disambiguating similar object-place paired associates. However, it requires examining functions of other upstream structures of the hippocampus to conclusively assign the role of associating object and place information to the hippocampus. If, for example, lesions produced in the perirhinal cortex produce similar deficits, it would be premature to conclude that the association between object and place information uniquely occurs in the hippocampus.

To elucidate the relative contributions of the MTL structures in the hippocampal-dependent object-place paired-associate task (Fig. 1), we manipulated the perirhinal cortex in the current study, one of the regions implicated as an object-information provider to the hippocampus (Knierim et al. 2006; Eichenbaum and Lipton 2008). Here we tested whether the perirhinal cortex was involved in the acquisition of new object-place paired associations. Importantly, we also tested the perirhinal cortical contributions to retrieving learned paired associates between objects and places. In the current study, the rats needed to pay attention to both object and place information. Therefore, if the perirhinal cortex is unique in its function for providing object information to the hippocampus, it is predicted that lesions in the perirhinal cortex will produce severe deficits as seen in the hippocampal lesioned animals in our previous study. A simple object-discrimination task that did not require spatial information was also employed to further examine the role of the perirhinal cortex only in specific conditions.

\section{Results}

NMDA-induced lesions in the perirhinal cortex

NMDA was used for producing axon-sparing lesions in the perirhinal cortex bilaterally. Figure 2A,B shows that NMDA infusions in the 
A.

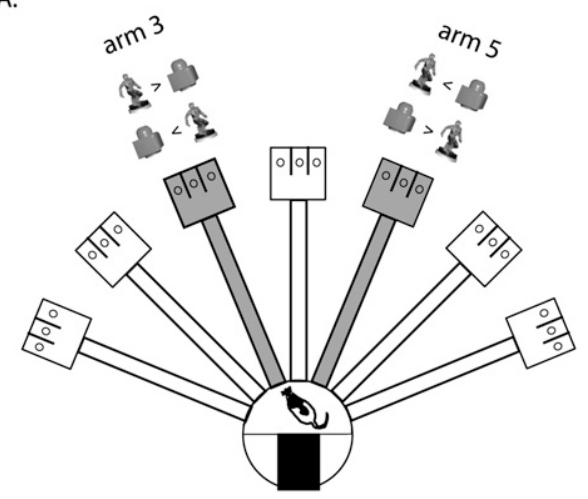

B.
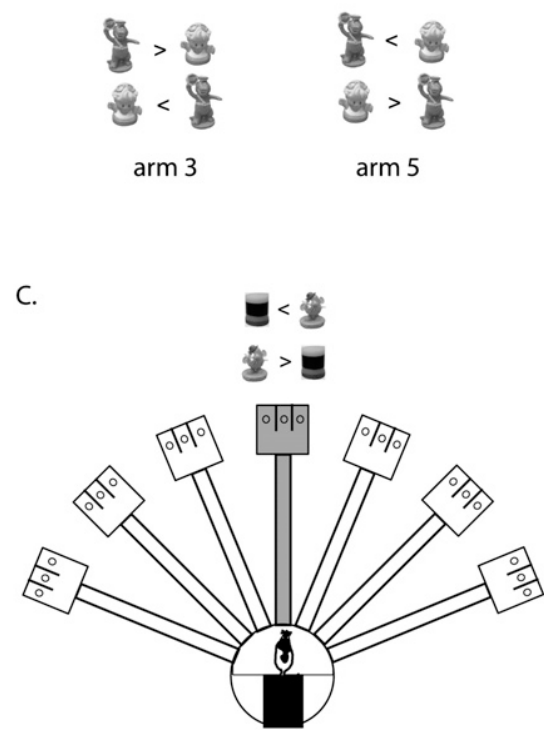

Figure 1. Illustration of the radial arm maze and behavioral paradigms. (A) Phase 1: Two objects (Spider-Man and LEGO block) were presented on arms 3 and 5 in gray color. Only one of the objects was rewarded in arm 3 (Spider-Man) and arm 5 (LEGO block) irrespective of its locations in the choice platform. Possible configuration of objects and appropriate choices are provided for both arms. In each trial, only one arm was open in the maze and objects were available in that open arm. (B) Phase 2: For acquisition of novel object-place paired associations, a pair of new objects (Barney and Girl) was presented on arms 3 and 5. Possible locations of the objects are shown as in $A$. Each object was rewarded only in a particular arm (Barney in arm 3 and Girl in arm 5) irrespective of its location in the choice platform. (C) Phase 3: Illustration of the task using only one arm (arm 4) in the maze. Two new objects (Mr. Potatohead and Cylinder) were used and the Mr. Potatohead choice was rewarded regardless of its location in the choice platform.

perirhinal cortex produced extensive damage along the rhinal fissure compared to the saline infusions. However, the rostral parts of the perirhinal cortex (from -7.3 to $-7.5 \mathrm{~mm}$ posterior to bregma) were spared in some rats relative to its anterior parts (Fig. 2B). The adjacent cortical areas were largely intact in the perirhinal lesion group (PR-LES). The myelin-stained sections showed that the perirhinal cortex lacked myelination and axons in the adjacent cortices were not damaged by NMDA (Fig. 2A). The volumetry analysis revealed that the percent of damage to the perirhinal cortex in the PR-LES group was $73.2 \pm 3.6 \%$ (mean \pm SEM) relative to the control group (PR-CTRL). Bilateral damage to the adjacent entorhinal cortex was also analyzed and the mean bilateral damage to the entorhinal cortex in the PR-LES group was $2.6 \pm$ $1.5 \%$, compared to the PR-CTRL group. The lesions were, therefore, fairly localized in the perirhinal cortex.

\section{Results from behavioral experiments}

In the current study, the same animals underwent three different phases of behavioral testing after surgery as illustrated in Figure 1 (see Materials and Methods for details) and behavioral results are provided following the behavioral testing schedule.

\section{Phase I: Effects of the perirhinal cortical lesions on retrieving object-place paired-associate memory}

Both groups of rats learned the task well prior to surgery, showing $\sim 90 \%$ correct performance on the last day of acquisition (Fig. $3 \mathrm{~A})$. Across $8 \mathrm{~d}$ of postsurgical testing, the PR-CTRL group maintained over $80 \%$ correct performance from the beginning. The PRLES group, in contrast, exhibited less than 65\% correct performance during the first two days but gradually improved their performance to the control level. An ANOVA with a repeatedmeasures design showed significant effects of the lesion group $\left(F_{(1,10)}=14.4, P<0.01\right)$ and postsurgical day $\left(F_{(7,70)}=19.25, P<\right.$ 0.001 ), as well as a significant interaction between the two variables $\left(F_{(7,70)}=7.22, P<0.001\right)$. A post-hoc pairwise comparison (Bonferroni $t$-test) demonstrated that there were significant differences in correct performance between the groups in days 1-4 $(P$-values $<0.05)$, whereas no difference was observed in days 5-8 $(P$-values $>0.1)$.

\section{Phase 2: Effects of the perirhinal cortical lesions on learning new object-place paired associations}

After the $8 \mathrm{~d}$ of postsurgical testing (Fig. 3A), a pair of new objects (Barney and Girl) were presented in the same arms of the maze for $10 \mathrm{~d}$ (Fig. 1B). This manipulation thus required the rats to associate novel objects with familiar places. During the acquisition period of $10 \mathrm{~d}$, the PR-CTRL and PR-LES groups exhibited marked differences in the learning curves (Fig. 3B). Specifically, both groups started at chance level on day 1 when the new objects were first introduced. The PR-CTRL group, however, showed a slow (yet steady) improvement up to day 4 and then exhibited a steep increase in performance between days 4 and 5, whereas the PR-LES group showed $\sim 50 \%-65 \%$ correct performance throughout the $10 \mathrm{~d}$ of testing period. An ANOVA with repeated measures revealed significant effects of the lesion group $\left(F_{(1,10)}=18.7, P=0.001\right)$, acquisition day $\left(F_{(9,90)}=12.5, P<0.001\right)$, and an interaction between the two variables $\left(F_{(9,90)}=6.18, P<0.001\right)$. A post-hoc paired comparison (Bonferroni $t$-test) demonstrated that the performance of the PR-CTRL was significantly higher than that of the PR-LES from day 4 onward ( $P$-values $<0.05)$, whereas there were no differences between the groups in days $1-3$ ( $P$-values $>0.1)$.

\section{Response bias}

When the rats failed to learn the current object-place pairedassociate task, they chose a food well on a particular side of the choice platform (e.g., left food well) regardless of the object occupying the food well (Lee and Solivan 2008). To measure this bias, a response bias index was calculated per day by taking an absolute value from the following formula: (\#Left Choice - \#Right Choice)/ (\#Left Choice + \#Right Choice), where \#Left Choice or \#Right Choice indicates the number of left or right choices made in the task. Thus, a response bias index of 1 indicates that the rat only chose the left (or right) food well throughout 32 trials and an index of 0 represents equal proportions of left and right food-well choices.

During the postsurgical days $1-8$, the PR-CTRL group consistently showed minimal response bias (Fig. 4A). However, the response bias of the PR-LES group started at a high level and gradually 
A

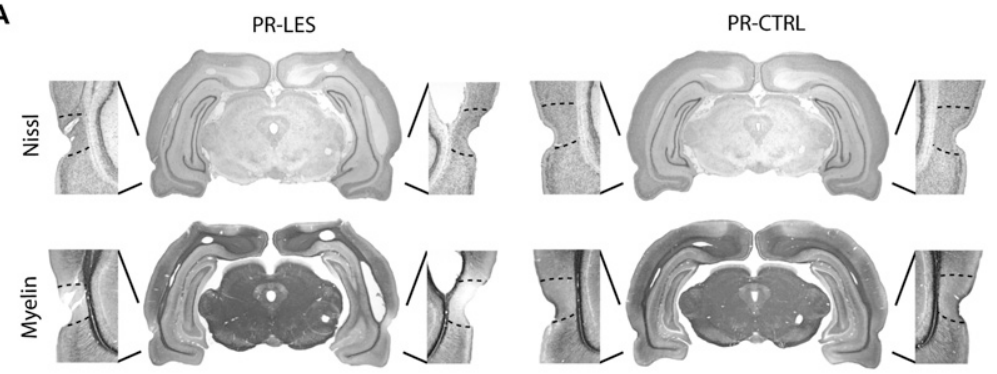

B

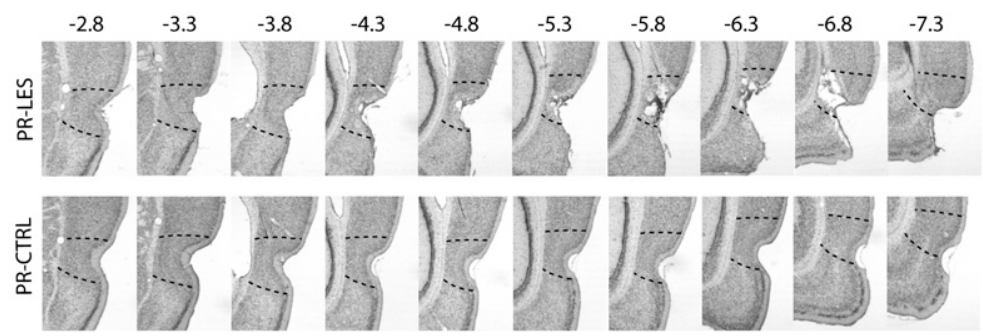

Figure 2. Histological verifications of perirhinal cortical lesions. (A) Representative sections from the PR-LES and PR-CTRL groups. The upper panel shows Nissl-stained sections and the lower panel shows the adjacent sections stained for myelin. (B) Serial, Nissl-stained sections presented from anterior to posterior directions (from -2.8 to $-7.3 \mathrm{~mm}$ from bregma).

decreased to control level as the lesioned rats relearned the objectplace paired associations. A repeated-measures ANOVA revealed significant effects of the lesion group $\left(F_{(1,10)}=7.5, P<0.05\right)$, postsurgical day $\left(F_{(7,70)}=6.2, P<0.001\right)$, and the interaction between the two variables $\left(F_{(7,70)}=2.28, P<0.05\right)$. A post-hoc paired comparison (Bonferroni $t$-test) showed that there was a significant difference in response bias between the groups on day $1(P<0.05)$. No difference was observed between the groups from day 2 onward ( $P$-values $>0.08$ ).

As new objects were introduced after $8 \mathrm{~d}$ of testing with familiar objects, the response bias of the PR-CTRL group stayed at a relatively high level initially but dropped sharply on the fifth day as the object-in-place strategy of the task was learned (Fig. 4B). In contrast, the response bias of the PR-LES group increased constantly throughout the learning period (except for the last three days). An ANOVA with a repeated-measures design showed significant effects of the lesion group $\left(F_{(1,10)}=23.7, P=0.001\right)$ and the interaction between the lesion group and postsurgical day $\left(F_{(9,90)}=\right.$ $3.44, P=0.001)$. There was no significant effect of the postsurgical day $\left(F_{(9,90)}=1.58, P>0.1\right)$. A post-hoc paired comparison (Bonferroni $t$-test) showed that the response bias of the PR-CTRL was significantly higher than that of the PR-LES from day 5 onward $(P$-values $<0.05)$. However, no difference was found between the groups until day 4 ( $P$-values $>0.1)$.

\section{Choice latency}

To examine the possibility of generic sensory-motor deficits associated with the PR-LES group, the latency to displacing an object in the choice platform from exiting the start box was measured in each trial. During the postsurgical testing period when the rats were tested with the familiar objects, the latency of the PR-LES group was compatible with that of the PR-CTRL group (Fig. 5A). An ANOVA on the latency averaged across $8 \mathrm{~d}$ showed no significant difference between the groups $\left(F_{(1,10)}=2.61, P>0.1\right)$. Again, no difference was found in the latency averaged across $10 \mathrm{~d}$ between the groups $\left(F_{(1,10)}=0.8, P>0.3\right)$ when they were further tested with the novel objects (Fig. 5B).
Phase 3: Simple object-discrimination task

The same animals were then given a simple object-discrimination task (Fig. 1C). The rats were required to choose one of two novel toy objects (Mr. Potatohead and a Cylinder) in arm 4 (center arm) regardless of the food-well locations associated with the objects in the choice platform. The Mr. Potatohead object was always rewarded. In this task, therefore, it was unnecessary to bind object and spatial (or arm) information because the spatial variable (i.e., arm 4) remained constant throughout the task.

As shown in our previous study (Lee and Solivan 2008), rats in both groups learned the simple object-discrimination task very quickly and showed over $70 \%$ correct performance from the onset of the training period (Fig. 6). A repeatedmeasures ANOVA revealed a significant effect of postsurgical day $\left(F_{(2,20)}=69.3\right.$, $P<0.001)$. However, there were no effects of the lesion group $\left(F_{(1,10)}=1.2, P>0.2\right)$ and the interaction between the lesion group and postsurgical day $\left(F_{(2,20)}=1.14\right.$, $P>0.3)$. These results suggest that the rats with the perirhinal cortical lesions were able to discriminate objects normally as long as either no association is required with space or no ambiguity exists in object-place paired associates to be learned, or both. The data also show that the rats were fully motivated for reward in the current study.

A

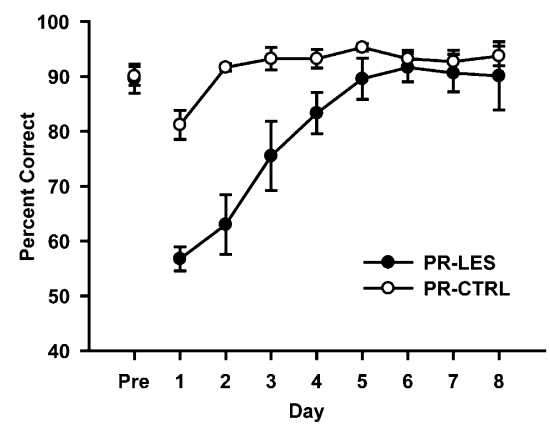

B

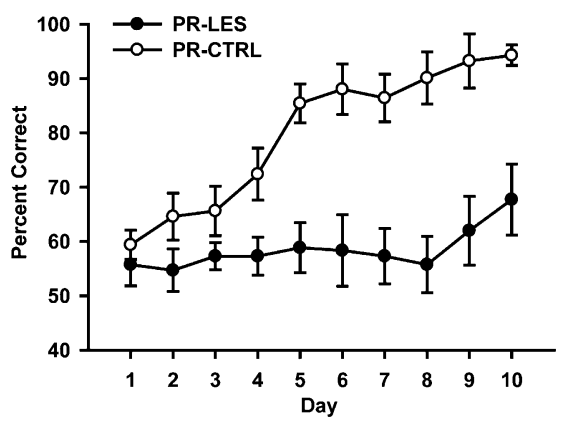

Figure 3. Effects of perirhinal cortical lesions on behavioral performance. $(A)$ Effects of the perirhinal cortical lesions on previously acquired paired-associative memory. Mean \pm SEM. (B) Acquisition curves for $10 \mathrm{~d}$ after introducing the new objects. Mean \pm SEM. 
A

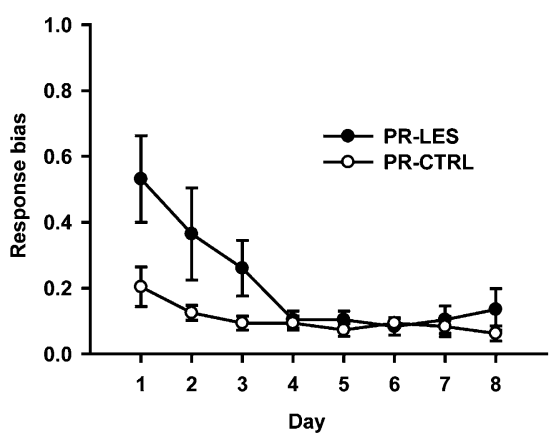

B

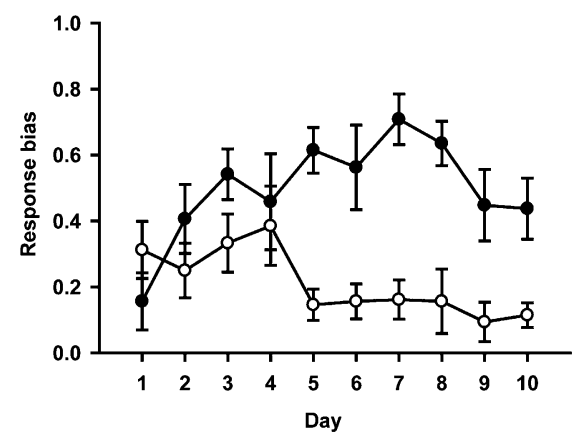

Figure 4. Response biases during behavioral testing. ( $A$ ) Response bias index $(0=$ no response bias; $1=$ complete response bias $)$ calculated across $8 \mathrm{~d}$ of testing for previously acquired paired associates. (B) Response bias index during $10 \mathrm{~d}$ of testing for the acquisition of novel object-place paired associates. Mean \pm SEM.

\section{Discussion}

The current study examined the effects of the perirhinal cortical lesions on retrieving and acquiring object-place paired associations. Compared to the PR-CTRL group, the PR-LES group showed profound deficits initially in retrieving the paired-associate memories between familiar objects and places learned before surgery, but eventually relearned the task. When a pair of new objects was presented in the same places of the maze, the PR-LES group failed to form new paired associations between novel objects and familiar places throughout $10 \mathrm{~d}$. However, the perirhinal cortical lesions did not affect the acquisition of the simple object-discrimination task. These results provide compelling evidence that the perirhinal cortex is necessary only in specific conditions, such as acquiring novel object-place paired associations and/or disambiguating similar object-place paired associates.

Perirhinal cortical functions have been widely tested in visual recognition tasks (Meunier et al. 1993; Suzuki et al. 1993; Mumby and Pinel 1994; Buffalo et al. 1998; Murray and Bussey 1999; Brown and Aggleton 2001; Winters et al. 2004; Barker et al. 2007; Davies et al. 2007) and it appears that the perirhinal cortex is involved in this cognitive domain, especially when delays are introduced or when conditional reinforcement schedules are used with complex visual stimuli. It is important to note, however, that damage in the hippocampus produces relatively mild impairment, if any, in the majority of behavioral tasks used for testing the role of the perirhinal cortex (Aggleton et al. 1986; Murray and Mishkin 1998; Aggleton and Brown 1999; Mumby 2001; Winters et al. 2004). In addition, the perirhinal cortex is not involved in a purely spatial task (Ennaceur et al. 1996; Glenn and Mumby 1998; Bussey et al. 1999; Winters et al. 2004) (but see Abe et al. 2008), whereas the role of the hippocampus is well established in such a task. Since the perirhinal cortex has connections with the hippocampus directly or indirectly via the entorhinal cortex (Burwell and Amaral 1998; Naber et al. 1999), using a single behavioral paradigm dependent on both the perirhinal cortex and hippocampus should be a promising approach if one is interested in studying the functional interactions between the two structures. The current task meets this condition because it requires not only the recognition of objects and places, but also the discrimination between the representations of similar object-place paired associates. The hippocampal and perirhinal cortical lesioned rats both exhibit deficits in this task. Prior studies are in line with our results because they showed robust impairments when the interactions between the two regions were interrupted (by disrupting either structure) in object-in-place tasks (Gaffan and Parker 1996; Bussey et al. 2000, 2001; Lee et al. 2005; Barker et al. 2007; Bachevalier and Nemanic 2008; Lee and Solivan 2008).

A previous study (Bussey et al. 2001) examined the role of the perirhinal cortex in a biconditional task similar to ours, using a Y-maze. Some commonalities in the results between that study and the current study are noticeable. In the study by Bussey et al. (2001), for example, perirhinal cortical lesions also prevented the rats from learning new paired associations between a pair of objects and a pair of places. The same lesions, however, produced minimal deficits in simple object discrimination using a single pair of objects. The current study corroborates these results. In addition, our study adds novel information and demonstrates that the lack of the perirhinal cortical function can be compensated when previously learned associations between familiar objects and places need to be discriminately retrieved. As shown in Figure 2B, the PRLES group was initially impaired in performing the familiar task, which suggests that the perirhinal cortex was normally involved in retrieving familiar object-place paired associations. However, it appears that normal learning (or relearning) occurred in the absence of the perirhinal cortex as the learning curve of the PR-LES group (Fig. 3A) was similar to the normal acquisition curve of the PR-CTRL in the novel object-place paired-associative learning (Fig. 3B). It is possible that the retrieval deficits were rescued by partially remaining perirhinal cortex. Other possibilities include that other structures, such as prefrontal cortex, parietal cortex, or lateral entorhinal cortex may have compensated for the lack of the perirhinal cortex in the PR-LES group (Kesner and Ragozzino 2003; Rogers and Kesner 2003; Hargreaves et al. 2005).

As shown in both the Bussey et al. (2001) study and our study, however, the initial acquisition of novel associations between objects and places required the perirhinal cortex. Although the PR-LES group showed some minimal signs of improvement in the
A

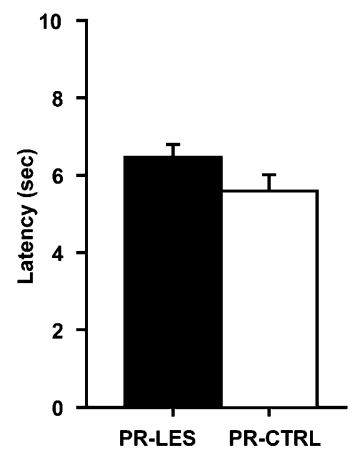

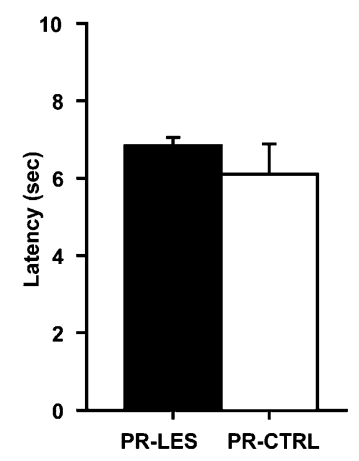

Figure 5. Choice latency. $(A)$ Average latency to choosing an object across $8 \mathrm{~d}$ of testing. $(B)$ Average latency during $10 \mathrm{~d}$ of acquisition of novel object-place paired associates. Mean \pm SEM. 


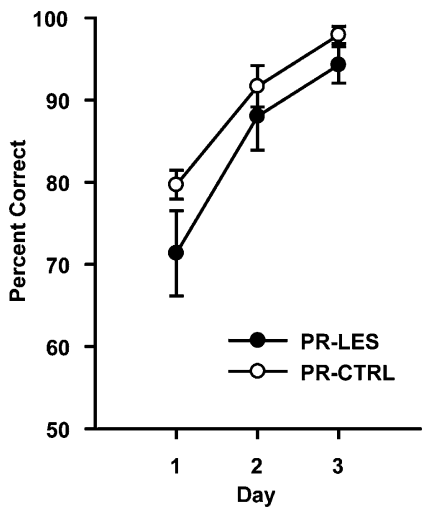

Figure 6. Acquisition of simple object-discrimination task. Learning curves across $3 \mathrm{~d}$. Mean \pm SEM.

last two days across $10 \mathrm{~d}$ of testing, not being able to improve their performance at all from chance level through eight consecutive days of testing strongly suggests that the perirhinal cortex is necessary for normal learning to take place when pairs of objects and places need to be associated and discriminated. Why is the perirhinal cortex required at the time of acquisition, but not during performance in the current task? Only some speculations can be provided here as follows: It implies that the perirhinal cortex performs a unique computation that may not be easily taken over by other regions when novel object-place pairs need to be initially associated. It is unlikely that the nature of computation is to provide simple sensory information (e.g., individual object's identity information) to the hippocampus because no deficit was observed in the simple object-discrimination task in the PR-LES group compared to the controls (Fig. 6). One candidate would be to disambiguate similar and complex visual scenes with objects embedded in those scenes. In our task, when the rat places itself in front of a pair of objects arranged in a certain configuration (e.g., a Spider-Man toy on the left and a LEGO block on the right), it is likely that those objects are perceived with the background scenes associated with a given arm. Since the locations of the objects in a given platform were counterbalanced and they were always presented simultaneously, there were four possible object configuration-scene combinations theoretically. It would be a reasonable assumption that the similarities among those combinatorial representations were fairly high because the same objects and scenes (associated with two different arms) were repeatedly used throughout the task.

Supporting this line of reasoning, the role of the perirhinal cortex in configural or scene learning (Buckley and Gaffan 1998; Eacott et al. 2001; Gaffan et al. 2004; Haskins et al. 2008), as well as in discriminating overlapping, thus ambiguous configural stimuli (Buckley and Gaffan 1997; Eacott et al. 2001; Bussey and Saksida 2002; Bussey et al. 2003) has been suggested in prior studies. It is possible that the successful establishment of discrete representations out of ambiguous configural scenes (involving objects) in the perirhinal cortex is a prerequisite for the presumed associative function of the hippocampus for binding those discrete representations with spatial information for constructing a cognitive map of the environment. Our data suggest that the above-proposed interaction between the perirhinal cortex and the hippocampus is particularly essential during the initial acquisition of object-place paired association. Once acquired through the perirhinal cortex, however, our results suggest that other brain regions may provide similar representations to the hippocampus.

Producing discrete and discriminative responses to similar events in space should involve multiple brain regions interacting with each other as a unified functional network. The conditional object-place paired-associate task used in the current study can serve as a powerful paradigm for investigating unique contributions of different regions, as well as the interactions among them. For example, the medial prefrontal cortex is as important as the hippocampus in the current paradigm (Lee and Solivan 2008), and the perirhinal cortex is also important as reported here. Simultaneous recording of these critical brain areas using this common behavioral framework will shed light on the neural mechanisms of how events are represented and utilized in the brain in the future.

\section{Materials and Methods}

\section{Subjects}

Twelve male Long-Evans rats weighing 310-330 g at the start of the experiment were housed individually in standard rodent cages. Animals were maintained on a $12 \mathrm{~h}$ light/dark cycle (lights on at 7:00 a.m.). All behavioral experiments were performed during the light phase of the cycle. Each rat had unlimited access to water, but was food-deprived to $80 \%$ of its free-feeding weight for behavioral testing.

\section{Behavioral apparatus}

Detailed descriptions of the behavioral apparatus can be found in our previous study (Lee and Solivan 2008). Briefly, a modified radial arm maze was used throughout the experiment (Fig. 1). The maze was located in the center of a testing room and surrounded by black curtains, which contained several distinctive visual cues. A start box with an opaque guillotine door was located in a circular center stage, from which seven arms $(10 \times 80 \mathrm{~cm}$ each, separated by $25.7^{\circ}$ from each other) radiated outward. The distal end of each arm was connected to a rectangular choice platform containing three food wells separated from each other by transparent vertical dividers. A transparent guillotine door was available at the entrance of each arm to allow access to the arm. There was a CCD camera above the maze for recording behavioral experiments. Two loud speakers were placed underneath the center stage to provide white noise during the behavioral experiments.

\section{Object-place paired-associate task}

Detailed procedures were provided in a previous study (Lee and Solivan 2008). Briefly, all rats were first familiarized with general procedures of the task for $\sim 1-2 \mathrm{wk}$. The rat started the object-place paired-associate task once it finished 32 trials of a simple food retrieval task within $30 \mathrm{~min}$. Once the training for the main task started, two arms (arms 3 and 5, which were the third and fifth arms from the left clockwise; Fig. 1) of the maze were used in the object-place paired-associate task. In each trial, only one of the two arms was open and two food wells (left and right food wells; the middle food well was never used in the task) of the choice platform in the open arm were completely covered by two toy objects (Spider-Man and a LEGO block, 7 and $6 \mathrm{~cm}$ tall, respectively). The objects used throughout in the current study were equally explored and no innate preference for either object was observed. Upon release from the start box, the rat entered one of the two arms and encountered two objects in the choice platform. To obtain a cereal reward, the rat should learn to displace a particular object (SpiderMan in arm 3 and the LEGO block in arm 5) irrespective of the object's location (left or right food well) within the choice platform. The locations of the toy objects in a given choice platform were counterbalanced across trials. Thus, the current object-place paired-associate task requires the rat to associate a particular place in the maze (arm 3 or arm 5) with certain object information (LEGO block or Spider-Man). When the rat made a correct choice and retrieved a food reward, the experimenter gently guided the animal to the start box with a small Plexiglas panel. While the rat consumed the reward, the experimenter prepared for the next trial. A wrong choice in the choice platform was punished by preventing the rat from correcting its response toward the baited food well and guiding the animal to the start box with no food reward. 
Thirty-two trials were given per day with an intertrial interval of 20-40 sec between trials. The sequence of visiting different arms was pseudorandomized and two different sequences were alternately used between days. The latency from entering an arm to displacing an object in the choice platform was measured offline. After being trained to criterion $(\geq 75 \%$ correct choices for both arms for two consecutive days), each rat received surgery (see below). It took $7-13 \mathrm{~d}$ (median $=9 \mathrm{~d}$ ) for the rats to learn the task to criterion before surgery.

\section{Surgery}

Animals were randomly assigned to one of two groups (PR-LES, $n=6$; PR-CTRL, $n=6$ ). Each rat was first anesthetized with isoflurane in an induction chamber, followed by intraperitoneal injection of Ketamine $(60 \mathrm{mg} / \mathrm{kg})$ and Xylazine $(8 \mathrm{mg} / \mathrm{kg})$. The animal was placed in a stereotaxic instrument (Stoelting). The anesthesia was maintained by isoflurane afterward throughout surgery. The scalp was incised, and the skull was adjusted to place bregma and lambda on the same horizontal plane. Small burr holes were drilled in the skull for excitotoxic lesions in the perirhinal cortex. A stainlesssteel cannula (30 gauge) was angled at $20^{\circ}$ from a vertical midline with the tip oriented laterally and lowered bilaterally into the brain using the following coordinates (presented in the following order: posterior to bregma, lateral to midline, and ventral from skull surface): (1) $3.3,3.5$, and $8.1 \mathrm{~mm}$, (2) $4.3,3.9$, and $8.0 \mathrm{~mm}$, (3) $5.3,4.6$, and $7.4 \mathrm{~mm}$, (4) $6.3,4.1$, and $7.6 \mathrm{~mm}$, and (5) 7.3, 4.0, and $6.7 \mathrm{~mm}$. Axon-sparing lesions were made with $N$-methyl-D-aspartate (NMDA) injections $(20 \mathrm{mg} / \mathrm{mL}$ and $0.05 \mu \mathrm{L}$ per site at $10 \mu \mathrm{L} / \mathrm{h}$ ) in the PR-LES group using a $10-\mu \mathrm{L}$ Hamilton syringe (Hamilton) operated by a microinjection pump (KD Scientific). The cannula was left in place $1 \mathrm{~min}$ before and after each injection to ensure proper diffusion of the neurotoxin in the target site. The PR-CTRL group received the same surgical procedures with an exception that equivalent volumes of phosphate-buffered saline (PBS) were infused into the perirhinal cortex. All protocols conformed to the NIH Guide for the Care and Use of Laboratory Animals and the Institutional Animal Care and Use Committee.

\section{Behavioral testing after surgery}

The rats were given $1 \mathrm{wk}$ for recovery and then were tested for $8 \mathrm{~d}$ (days 1-8) in the same object-place paired-associate task (Phase 1 , Fig. 1A). This was to investigate the effects of the perirhinal cortical lesions on the retrieval of the object-place paired-associate memories acquired prior to surgery. From day 9 (Phase 2), a new pair of toy objects (Barney and Girl, 8 and $5 \mathrm{~cm}$ tall, respectively) was introduced for $10 \mathrm{~d}$ (days 9-18) in the same arms used for the previous testing (Fig. 1B). This was to examine the effects of the perirhinal cortical lesions on the acquisition of new object-place paired associations. The task was identical with its previous version, except that the animals were required to associate new objects with the familiar locations (Barney was rewarded in arm 3 and a Girl toy object was rewarded in arm 5). Experimenters were blind to the surgical manipulations of the animals throughout the study.

\section{Simple object-discrimination task}

After finishing the object-place paired-associate task, the rats were finally tested with a simple object-discrimination task for $3 \mathrm{~d}$ (Phase 3; days 19-21, Fig. 1C). This was to test the involvement of the perirhinal cortex in a situation where objects need to be discriminated from each other independent of their associated spatial locations. To eliminate the spatial component, a single arm (i.e., arm 4) that had not been visited for the previous object-place paired-associate task was used. When the start box door was opened, the rat reached the choice platform and chose one of two new objects (Mr. Potatohead and Cylinder, 5 and $4 \mathrm{~cm}$ tall, respectively). The Mr. Potatohead object choice was always rewarded. Thirty-two trials were given per day.

\section{Histology}

Histological verifications of lesions were performed after the completion of all behavioral experiments. All rats received a lethal dose of sodium pentobarbital (Euthasol, Virbac AH, Inc.) and were transcardially perfused with $0.9 \%$ saline, followed by a $10 \%$ formaldehyde solution. After extraction, the brains were stored in a $10 \%$ formalin- $30 \%$ sucrose solution at $4^{\circ} \mathrm{C}$ for $72 \mathrm{~h}$. The brains were frozen, cut into $40 \mu \mathrm{m}$ coronal sections on a sliding microtome (Microm HM 430, Thermo Fisher Scientific). Two series of every third section were collected on gelatin-coated slides. One set of sections was stained for Nissl with thionin (Sigma) and the other set was stained for myelin with gold chloride (Sigma), respectively. To quantify the amount of NMDA-produced damage in the perirhinal cortex (PR) and entorhinal cortex (EC) (from -2.8 to $-7.5 \mathrm{~mm}$ posterior to bregma), adjacent Nissl- and myelinstained sections were digitally photomicrographed and superimposed using Photoshop CS2 (Adobe Systems). Based on the myelin-stained section, the perirhinal cortical boundaries were drawn as a new image layer with a pen tablet as an input device. The superimposed, Nissl- and myelin-stained tissues made the delineation of the perirhinal cortical boundaries easier since the absence of myelination is a distinctive characteristic of the perirhinal cortex (Burwell 2001; Kholodar-Smith et al. 2008). Using the Nissl-stained section, the borders of the EC were also added into the new image layer, and the intact cell boundaries were delineated. The two-dimensional image layer of the undamaged cell area was converted to black-and-white bitmap format and stored as a separate image from the two stained images. The series of bitmap images for each rat was imported into a commercial software package (Voxwin 1.2.2, Voxar Co.) for three-dimensional reconstruction and volumetry (Lee and Solivan 2008). The percent damage to the PR and EC was calculated by comparing the average number of voxels used for reconstructing each brain area between the PR-LES and PR-CTRL groups.

\section{Acknowledgments}

The current study was supported by NIMH RO1 MH079971 and by the WCU program of the Ministry of Education, Science and Technology in Korea through KOSEF (R32-10142).

\section{References}

Abe H, Ishida Y, Nonaka H, Iwasaki T. 2008. Functional difference between rat perirhinal cortex and hippocampus in object and place discrimination tasks. Behav Brain Res 197: 388-397.

Aggleton JP, Brown MW. 1999. Episodic memory, amnesia, and the hippocampal-anterior thalamic axis. Behav Brain Sci 22: 425-489.

Aggleton JP, Hunt PR, Rawlins JN. 1986. The effects of hippocampal lesions upon spatial and non-spatial tests of working memory. Behav Brain Res 19: 133-146.

Bachevalier J, Nemanic S. 2008. Memory for spatial location and objectplace associations are differently processed by the hippocampal formation, parahippocampal areas $\mathrm{TH} / \mathrm{TF}$ and perirhinal cortex. Hippocampus 18: 64-80.

Barker GRI, Bird F, Alexander V, Warburton EC. 2007. Recognition memory for objects, place, and temporal order: A disconnection analysis of the role of the medial prefrontal cortex and perirhinal cortex. J Neurosci 27: 2948-2957.

Brown MW, Aggleton JP. 2001. Recognition memory: What are the roles of the perirhinal cortex and hippocampus? Nat Rev Neurosci 2: 51-61.

Buckley MJ, Gaffan D. 1997. Impairment of visual object-discrimination learning after perirhinal cortex ablation. Behav Neurosci 111: 467-475.

Buckley MJ, Gaffan D. 1998. Perirhinal cortex ablation impairs configural learning and paired-associate learning equally. Neuropsychologia 36: 535-546.

Buffalo EA, Reber PJ, Squire LR. 1998. The human perirhinal cortex and recognition memory. Hippocampus 8: 330-339.

Burwell RD. 2000. The parahippocampal region: Corticocortical connectivity. Ann N Y Acad Sci 911: 25-42.

Burwell RD. 2001. Borders and cytoarchitecture of the perirhinal and postrhinal cortices in the rat. I Comp Neurol 437: 17-41.

Burwell RD, Amaral DG. 1998. Cortical afferents of the perirhinal, postrhinal, and entorhinal cortices of the rat. J Comp Neurol 398: 179-205.

Bussey TJ, Saksida LM. 2002. The organization of visual object representations: A connectionist model of effects of lesions in perirhinal cortex. Eur J Neurosci 15: 355-364.

Bussey TJ, Muir JL, Aggleton JP. 1999. Functionally dissociating aspects of event memory: The effects of combined perirhinal and postrhinal 
cortex lesions on object and place memory in the rat. J Neurosci 19: 495502 .

Bussey TJ, Duck J, Muir JL, Aggleton JP. 2000. Distinct patterns of behavioural impairments resulting from fornix transection or neurotoxic lesions of the perirhinal and postrhinal cortices in the rat. Behav Brain Res 111: 187-202.

Bussey TJ, Dias R, Amin E, Muir JL, Aggleton JP. 2001. Perirhinal cortex and place-object conditional learning in the rat. Behav Neurosci 115: 776785.

Bussey TJ, Saksida LM, Murray EA. 2003. Impairments in visual discrimination after perirhinal cortex lesions: Testing "declarative" vs. "perceptual-mnemonic" views of perirhinal cortex function. Eur J Neurosci 17: 649-660.

Cahusac PM, Miyashita Y, Rolls ET. 1989. Responses of hippocampal formation neurons in the monkey related to delayed spatial response and object-place memory tasks. Behav Brain Res 33: 229-240.

Davachi L. 2006. Item, context and relational episodic encoding in humans. Curr Opin Neurobiol 16: 693-700.

Davies M, Machin PE, Sanderson DJ, Pearce JM, Aggleton JP. 2007. Neurotoxic lesions of the rat perirhinal and postrhinal cortices and their impact on biconditional visual discrimination tasks. Behav Brain Res 176: 274-283.

Eacott MJ, Machin PE, Gaffan EA. 2001. Elemental and configural visual discrimination learning following lesions to perirhinal cortex in the rat. Behav Brain Res 124: 55-70.

Eichenbaum H, Lipton PA. 2008. Towards a functional organization of the medial temporal lobe memory system: Role of the parahippocampal and medial entorhinal cortical areas. Hippocampus 18: 1314-1324.

Ennaceur A, Neave N, Aggleton JP. 1996. Neurotoxic lesions of the perirhinal cortex do not mimic the behavioural effects of fornix transection in the rat. Behav Brain Res 80: 9-25.

Furtak SC, Wei SM, Agster KL, Burwell RD. 2007. Functional neuroanatomy of the parahippocampal region in the rat: The perirhinal and postrhinal cortices. Hippocampus 17: 709-722.

Fyhn M, Molden S, Witter MP, Moser EI, Moser MB. 2004. Spatial representation in the entorhinal cortex. Science 305: 1258-1264.

Gaffan D. 1994. Scene-specific memory for objects: A model of episodic memory impairment in monkeys with fornix transection. J Cogn Neurosci 6: 305-320.

Gaffan D, Parker A. 1996. Interaction of perirhinal cortex with the fornixfimbria: Memory for objects and "object-in-place" memory. J Neurosci 16: $5864-5869$.

Gaffan EA, Healey AN, Eacott MJ. 2004. Objects and positions in visual scenes: Effects of perirhinal and postrhinal cortex lesions in the rat. Behav Neurosci 118: 992-1010.

Gilbert PE, Kesner RP. 2003. Localization of function within the dorsal hippocampus: The role of the CA3 subregion in paired-associate learning. Behav Neurosci 117: 1385-1394.

Gilbert PE, Kesner RP. 2004. Memory for objects and their locations: The role of the hippocampus in retention of object-place associations. Neurobiol Learn Mem 81: 39-45.

Glenn MJ, Mumby DG. 1998. Place memory is intact in rats with perirhinal cortex lesions. Behav Neurosci 112: 1353-1365.

Hafting T, Fyhn M, Molden S, Moser MB, Moser EI. 2005. Microstructure of a spatial map in the entorhinal cortex. Nature 436: 801-806.

Hargreaves EL, Rao G, Lee I, Knierim JJ. 2005. Major dissociation between medial and lateral entorhinal input to dorsal hippocampus. Science 308: 1792-1794.

Haskins AL, Yonelinas AP, Quamme JR, Ranganath C. 2008. Perirhinal cortex supports encoding and familiarity-based recognition of novel associations. Neuron 59: 554-560.

Kerr KM, Agster KL, Furtak SC, Burwell RD. 2007. Functional neuroanatomy of the parahippocampal region: The lateral and medial entorhinal areas. Hippocampus 17: 697-708.

Kesner RP, Ragozzino ME. 2003. The role of the prefrontal cortex in objectplace learning: A test of the attribute specificity model. Behav Brain Res 146: 159-165.
Kesner RP, Hunsaker MR, Warthen MW. 2008. The CA3 subregion of the hippocampus is critical for episodic memory processing by means of relational encoding in rats. Behav Neurosci 122: 1217-1225.

Kholodar-Smith DB, Boguszewski P, Brown TH. 2008. Auditory trace fear conditioning requires perirhinal cortex. Neurobiol Learn Mem 90: 537-543.

Knierim JJ, Lee I, Hargreaves EL. 2006. Hippocampal place cells: Parallel input streams, subregional processing, and implications for episodic memory. Hippocampus 16: 755-764.

Lee I, Solivan F. 2008. The roles of the medial prefrontal cortex and hippocampus in a spatial paired-association task. Learn Mem 15: 357-367.

Lee I, Hunsaker MR, Kesner RP. 2005. The role of hippocampal subregions in detecting spatial novelty. Behav Neurosci 119: 145-153.

Malkova L, Mishkin M. 2003. One-trial memory for object-place associations after separate lesions of hippocampus and posterior parahippocampal region in the monkey. J Neurosci 23: 1956-1965.

Meunier M, Bachevalier J, Mishkin M, Murray EA. 1993. Effects on visual recognition of combined and separate ablations of the entorhinal and perirhinal cortex in rhesus monkeys. J Neurosci 13: 5418-5432.

Mishkin M, Suzuki WA, Vargha-Khadem F, Gadian DG. 1997. Hierarchical organization of cognitive memory. Philos Trans R Soc Lond B Biol Sci 352: 1461-1467.

Mumby DG. 2001. Perspectives on object-recognition memory following hippocampal damage: Lessons from studies in rats. Behav Brain Res 127: $159-181$.

Mumby DG, Pinel JP. 1994. Rhinal cortex lesions and object recognition in rats. Behav Neurosci 108: 11-18.

Murray EA, Bussey TJ. 1999. Perceptual-mnemonic functions of the perirhinal cortex. Trends Cogn Sci 3: 142-151.

Murray EA, Mishkin M. 1998. Object recognition and location memory in monkeys with excitotoxic lesions of the amygdala and hippocampus. I Neurosci 18: 6568-6582.

Naber PA, Witter MP, Lopez da Silva FH. 1999. Perirhinal cortex input to the hippocampus in the rat: Evidence for parallel pathways, both direct and indirect. A combined physiological and anatomical study. Eur J Neurosci 11: 4119-4133.

Parkinson JK, Murray EA, Mishkin M. 1988. A selective mnemonic role for the hippocampus in monkeys: Memory for the location of objects. I Neurosci 8: 4159-4167.

Rogers JL, Kesner RP. 2003. Cholinergic modulation of the hippocampus during encoding and retrieval. Neurobiol Learn Mem 80: 332-342.

Smith ML, Milner B. 1981. The role of the right hippocampus in the recall of spatial location. Neuropsychologia 19: 781-793.

Stepankova K, Fenton AA, Pastalkova E, Kalina M, Bohbot Vo D. 2004. Object-location memory impairment in patients with thermal lesions to the right or left hippocampus. Neuropsychologia 42: 1017-1028.

Suzuki WA, Zola-Morgan S, Squire LR, Amaral DG. 1993. Lesions of the perirhinal and parahippocampal cortices in the monkey produce longlasting memory impairment in the visual and tactual modalities. I Neurosci 13: 2430-2451.

Suzuki WA, Miller EK, Desimone R. 1997. Object and place memory in the macaque entorhinal cortex. J Neurophysiol 78: 1062-1081.

Sziklas V, Lebel S, Petrides M. 1998. Conditional associative learning and the hippocampal system. Hippocampus 8: 131-137.

Vargha-Khadem F, Gadian DG, Watkins KE, Connelly A, Van Paesschen W, Mishkin M. 1997. Differential effects of early hippocampal pathology on episodic and semantic memory. Science 277: 376-380.

Winters BD, Forwood SE, Cowell RA, Saksida LM, Bussey TJ. 2004. Double dissociation between the effects of peri-postrhinal cortex and hippocampal lesions on tests of object recognition and spatial memory: Heterogeneity of function within the temporal lobe. J Neurosci 24: 5901-5908.

Witter MP, Amaral DG. 2004. The hippocampal formation. In The rat nervous system, 3rd ed. (ed. G Paxinos), pp. 635-704. Elsevier, Amsterdam, The Netherlands.

Received September 6, 2009; accepted in revised form November 30, 2009. 


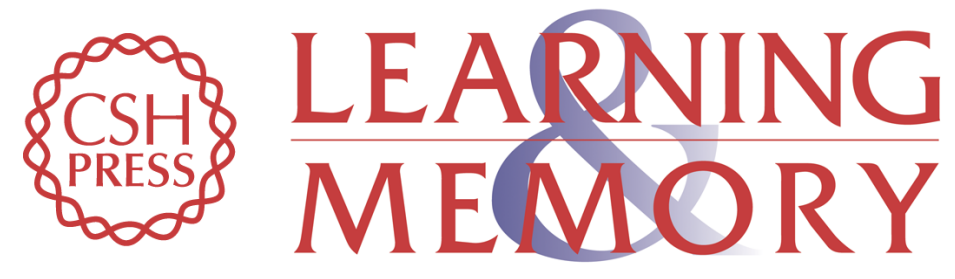

\section{Perirhinal cortex is necessary for acquiring, but not for retrieving object-place paired association}

Yong Sang Jo and Inah Lee

Learn. Mem. 2010, 17:

Access the most recent version at doi:10.1101//m.1620410

References This article cites 58 articles, 13 of which can be accessed free at:

http://learnmem.cshlp.org/content/17/2/97.full.htmI\#ref-list-1

License

Email Alerting Receive free email alerts when new articles cite this article - sign up in the box at the Service top right corner of the article or click here. 